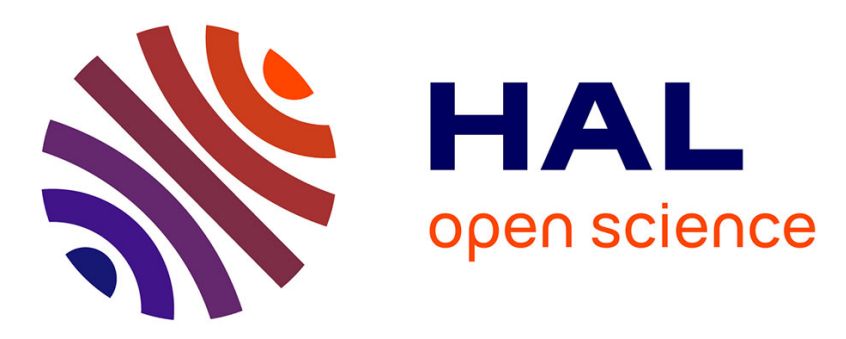

\title{
Rehabilitation Supervision Using Wireless Sensor Networks
}

\author{
Abdelkrim Hadjidj, Abdelmadjid Bouabdallah, Yacine Challal
}

\section{To cite this version:}

Abdelkrim Hadjidj, Abdelmadjid Bouabdallah, Yacine Challal. Rehabilitation Supervision Using Wireless Sensor Networks. IEEE International Symposium on a World of Wireless, Mobile and Multimedia Networks (WoWMoM), Jun 2011, Lucca, Italy. pp.1-3, 10.1109/WoWMoM.2011.5986123 . hal-00648818

\section{HAL Id: hal-00648818 https://hal.science/hal-00648818}

Submitted on 6 Dec 2011

HAL is a multi-disciplinary open access archive for the deposit and dissemination of scientific research documents, whether they are published or not. The documents may come from teaching and research institutions in France or abroad, or from public or private research centers.
L'archive ouverte pluridisciplinaire HAL, est destinée au dépôt et à la diffusion de documents scientifiques de niveau recherche, publiés ou non, émanant des établissements d'enseignement et de recherche français ou étrangers, des laboratoires publics ou privés. 


\title{
Rehabilitation Supervision Using Wireless Sensor Networks
}

\author{
Abdelkrim Hadjidj, Abdelmadjid Bouabdallah, Yacine Challal \\ Universit de Technologie de Compiègne, HEUDIASYC UMR CNRS 6599 \\ BP 20529, Compiègne Cedex, France \\ \{ahadjidj, bouabdal, ychallal\}@utc.fr
}

\begin{abstract}
Wireless sensor networks are becoming a topic of great interest in medical applications generating a large amount of work in medical and computer research communities. In this line, several solutions have been proposed to provide unobtrusive, flexible and low cost systems for patient supervision. However, existing solutions can not be used in rehabilitation supervision because of the specific characteristics of this application. Indeed, new major challenges arise from the fact that each sensor node needs to continuously stream large volumes of data at a high rate to enable doctors to extract clinically relevant information. In addition, the placement of several adjacent sensor nodes on the body may cause serious interferences problems. In this work, we describe and demonstrate our wireless sensor network prototype for high-fidelity rehabilitation supervision. Our demonstration allows attendees to experience the potential of wireless sensor networks for enabling flexible and low cost rehabilitation monitoring systems. In order to alleviate transmission problems, we develop a novel communication protocol that meets the clinical requirements of rehabilitation supervision in terms of data quality and data rate.
\end{abstract}

Keywords: wireless sensor networks, rehabilitation, communication protocols.

\section{INTRODUCTION}

Rehabilitation is an exercises program with the objective of achieving a physical functioning level that allows patients to return to their initial motor capabilities after an accident or a surgery. Studies show an increase in functional recovery of patients with increased amount of exercises during rehabilitation [1]. However, intensive exercises program requires continuous supervision of patients and increases the load for therapists and medical staff. Human motion monitoring techniques, such as optical measurement systems and force plate, attracted significant interest in recent years due to their potential to automate the supervision of rehabilitation therapy. These systems acquire, in real time, data about the changes in body motion/position, and process the information to characterize the movements. Nevertheless, such systems require a complicated set-up procedure and are too costly to be used in ambulatory or in-home environments.

The advances of Micro Electro Mechanical Systems, wireless technology and medical sensors have boosted wireless sensor networks for medical applications. Wireless sensor networks can collect information about individuals' state and provide feedback to the user in real time. Thus, they make possible the development of low-cost, light-weight, unobtrusive and easy-to-use monitoring solutions for patient supervision.
However, these existing systems can not be used in rehabilitation supervision because of the specific characteristics of these applications. Indeed, to be able to generate an accurate report and feedback, the system needs several information (acceleration, angular velocity, muscle activity) from several nodes in real time. Hence, the sensor sampling and data transmission should be performed at high frequency. In addition, the placement of several adjacent sensor nodes on the body may cause serious interferences problems. As a result, the network performance is degraded and a new communication protocol is definitely needed.

There has been several research work on wireless sensor networks for rehabilitation supervision. A great deal of these works have focused on sensor/hardware design [2], platform/architecture design [3] or signal processing algorithms design [4] with little attention paid to network communications.

In this paper, we describe and demonstrate a wireless sensor network for high-fidelity rehabilitation supervision using offthe-shelf wireless sensor modules. In addition, we address the challenge of designing a novel communication protocol that meets the clinical requirements of our supervision application in terms of data quality and data rate.

\section{SYSTEM ARCHITECTURE}

In this paper, we propose a prototype of wireless sensor network for rehabilitation supervision having three components : a set of motion sensor nodes, a basestation and a system manager software as illustrated in figure 1. The motion sensor nodes are worn by the patient and measure motion data (acceleration and angular velocity) as the patient moves his limbs. Data collected by the motion sensor nodes is wirelessly sent to the basestation. The basestation then relays the data to the system manager running on a laptop. Finally, the system manager processes, saves and displays the received data at real-time. To enhance the ergonomics of our solution, we use elastic adjustable bracelets for easy sensor node fixing. These bracelets can be easily fixed with no or minimal help.

Once a sensor node installed on the patient and switched on, it automatically joins the network without any thirdparty intervention. This is guaranteed by our communication protocol that ensures seamless network formation and nodes' discovery. We describe the communication protocol in more details in the section 4. Each motion sensor node has an 


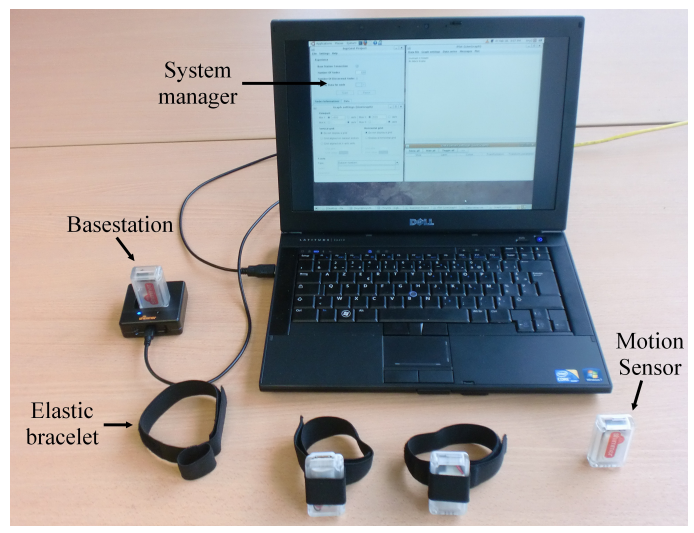

Fig. 1. System components

accelerometer and a gyroscope to measure patient's limb movements. It is preloaded with a TinyOS application that we developed to sample sensors and to send the collected motion data (acceleration and angular velocity in three axis) to the basestation. To reduce communication overhead and meet high frequency data sampling, a sensor node bundles several unit samples into a single message. Samples are temporarily stored in a sampling buffer and deleted after transmission.

The basestation acts as a network coordinator and forms a star topology together with motion sensor nodes. The basestation is a collection point for sensor nodes' data and it is also responsible for a number of functions such as node management, node synchronization and commands dissemination. The basestation is connected, through a USB cable, to a terminal station, such as a laptop, running the system manager. It runs a TinyOS application that acts as a bidirectional relay between the motion sensor nodes and the system manager. The basestation listens to the wireless medium and relays all packets coming from the network nodes to the system manager via the USB cable. Similarly, it listens to the USB port and broadcast commands coming from the system manager to all motion sensor nodes.

The data resulting from sensors sampling represent the voltage value of the sensor output at the moment of measurement. This value should be transformed into acceleration or angular velocity before being used by the backoffice software (such as a biomechanical model), that helps in patient control by doctors. In order to reduce energy consumption of sensor nodes, we proposed that sensor nodes send the raw data to the system manager which processes it into useful information. After data processing, the manager stores it in a clinical database for further signal processing and medical analysis. The data is used by doctors to check the patients' physical performance for the purpose of assessing the best therapies suitable for the patient. Since doctors may have no networking knowledge, we developed a simple user-friendly Java Graphical User Interface for the system manager application. The GUI enables doctors to start/stop/resume data collection, plot and filter downloaded data at real-time and access to previously recorded data for offline analysis.

\section{HARDWARE}

Our wireless sensor nodes are a light weight (15g), compact form factors $(50 \mathrm{~mm} \times 25 \mathrm{~mm} \times 12.5 \mathrm{~mm})$ and low power consumption sensor modules. These characteristics make them suitable for body worn clinical and in-home applications such as rehabilitation supervision. The core component of the sensor node is the Texas Instrument MSP $430 \mathrm{MCU}$ running at $8 \mathrm{MHz}$. This low power microcontroller has 10 KByte RAM, 48 KByte flash memory and an integrated 8channels 12-bits Analog to Digital Converter (ADC). Motion sensor nodes have a low-power MMA7260Q accelerometer with selectable sensitivity $(1.5 \mathrm{~g} / 2 \mathrm{~g} / 4 \mathrm{~g} / 6 \mathrm{~g})$. The MMA7260Q shows a low power consumption of $500 \mu \mathrm{A}$ in active mode against $3 \mu A$ in sleep mode. Another benefit of this accelerometer is the high sensitivity $(800 \mathrm{mv} / \mathrm{g})$ with the $1.5 \mathrm{~g}$ setting. Motion sensor nodes have also an add-on daughter board with an integrated InvenSence Dual-axis Gyroscope IDG300. The IDG300 offers a $500^{\circ} / \mathrm{sec}$ full scale range with a sensitivity of $2 \mathrm{mV} / \% / \mathrm{sec}$. We use $6 \mathrm{ADC}$ channels to capture the accelerometer and the gyroscope sensors data (1 channel per axis). To be able to communicate wirelessly, sensor nodes integrate the widely-used IEEE 802.15.4 compliant CC2420 radio-transceiver. This chipcon has a theoretical bandwidth of $250 \mathrm{kbps}$, a programmable output power and a hardware support for error correction.

\section{COMMUNICATION PROTOCOL}

We propose a polling communication protocol in which we adopt the star network topology to minimize collisions, synchronize nodes and reduce the energy consumption through low duty cycle. The basestation has a major role in this protocol and orchestrates the whole network. It periodically sends a control message to request all devices to send their data and to define the exact moment at which each node is expected to send the data to avoid collisions. Upon receiving this message, a node bundles all ready data into a packet and waits the instant at which it can send this packet. When the node is permitted to begin the transmission process, it sends its packets and waits the next control message. This functioning avoids the collision problem of contention access method.

The base station control message serves also as an advertisement for new nodes willing to join the network. Upon receiving this message, a node wishing to join the network sends an association request to the base station and waits for an acknowledgement. Upon receiving this association request, the base station allocates a new timeslot for this node and acknowledges the association request. Henceforth, the node is part of the network and can use its corresponding timeslot to transmit data.

The larger power consumption of our protocol comes from the need for a node to periodically wake up and listen to the control message. However, the protocol avoids, to the node, continuous listening to achieve synchronization or to access the channel. Indeed, the nodes knows exactly the moment at which it is permitted to transmit data and can achieve low duty-cycle by switching off the radio transceiver all the time 
except at its own time slots and expected time of each control message. As a result, the device conserves its energy and achieves longer lifetime even though data transmission rate is high. Since a timeslot is exclusively used by one node, data is sent without listening to the channel economizing power energy at the same time .

Although not desirable, occasionally loss packets is not critical for rehabilitation applications as long as missing packets are correctly identified. Indeed, because sensors sampling is performed at high frequency, a lost packet does not impact the accuracy of collected information and can be extrapolated from correctly received data. Wherefore, our communication protocol does not use acknowledgements for data packets. Likewise, each sent packet is identified by a sequential number in the "message ID" field to enable the supervision application to detect any missing packet.

\section{Demo Setup}

We will start our demonstration by presenting the different components of our prototype and their functions. Then, the motion sensor nodes, worn by a patient (figure 2), will be switched on and will join the network without any third-party intervention. The user-friendly interface of our system shows discovered sensor nodes at real time as illustrated in figure 3. The patient can start its exercises session, when all nodes have been discovered, by clicking on the start button. After this short setup process, the patient starts performing different tasks (walking, working on the computer, swinging the arm) while sensor nodes measure the acceleration and the angular velocity of different segments of its arm. The application receives the collected data wirelessly and plots it on various realtime graphs to show the performance and the high frequency of data collection (figure 3 ).

The participants of the conference will be able to wear our motion sensor nodes and see their kinematic measures when they perform some exercises. To illustrate details of our communication protocol, we will prepare a poster which describes the different mechanisms implemented. We believe this will arise the attention of participants and incite them to discuss our ideas. As a result, the presentation is more reactive and gives us a valuable feedback and directions for our future works.

\section{CONCLUSION}

In this paper we have presented a new wireless sensor network for high-fidelity rehabilitation supervision. This system is a low cost, light-weight and easy to use monitoring system that can be used in-home or within a hospital. Contrary to existing wireless sensor networks for rehabilitation, our solution relies on a novel communication protocol that meets the clinical requirements of rehabilitation supervision in terms of data quality and data rate. We have proved the functionality and the performance of our prototype by testing it in real environment.

Our project is a work in progress and much still remains to be done. In particular, we will replace the laptop by a lightweight PDA. This will bring an important added value

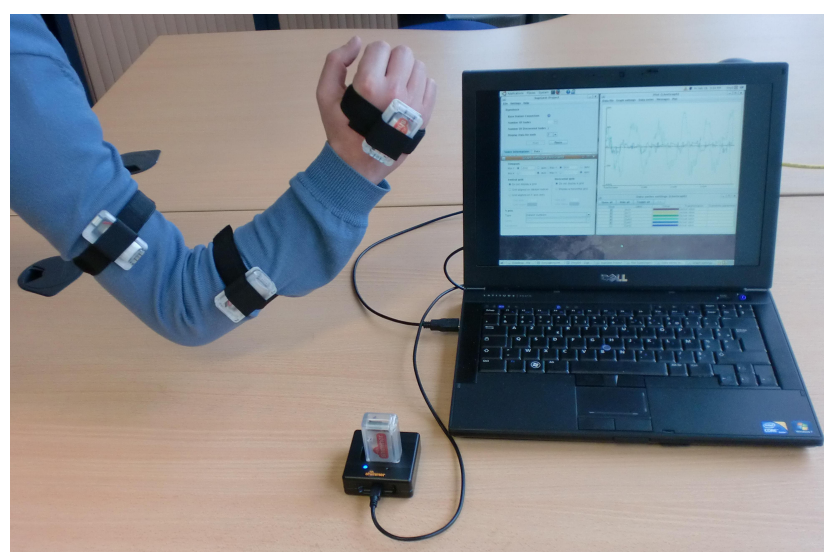

Fig. 2. Our prototype during lab experiment

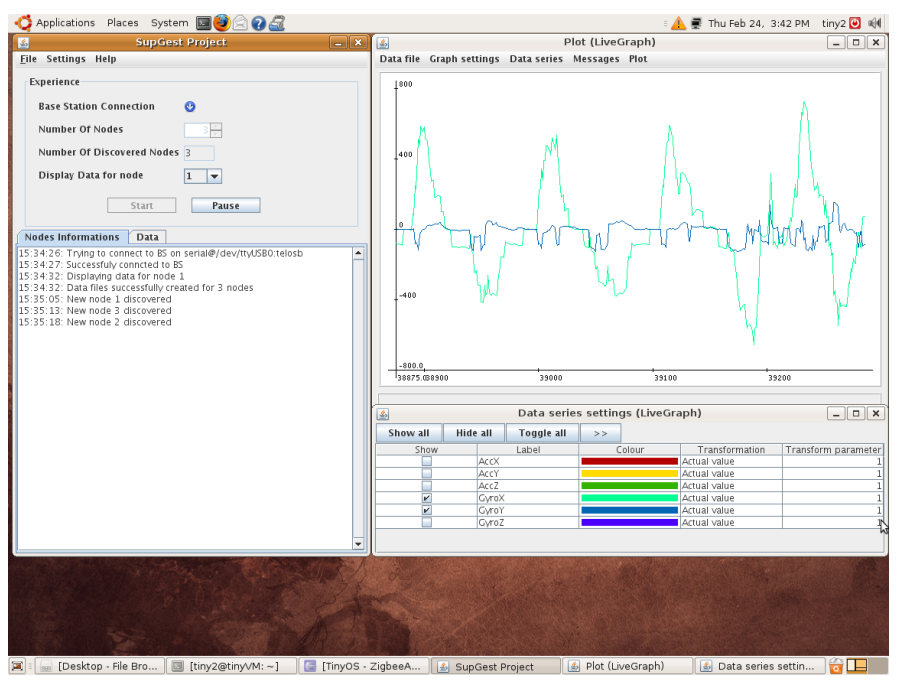

Fig. 3. Data collection snapshot

to our solution and improve the mobility and patient autonomy. Also, we plan to conduct more comprehensive tests in cooperation with doctors and physical therapists to provide a better evaluation of our solution. Finally, we need to tackle new challenges in order to incorporate our solution into a complete clinical information system.

\section{REFERENCES}

[1] G. Kwakkel, R. van Peppen, R. C. Wagenaar, S. W. Dauphinee, C. Richards, A. Ashburn, K. Miller, N. Lincoln, C. Partridge, I. Wellwood, and P. Langhorne, "Effects of augmented exercise therapy time after stroke: A Meta-Analysis," Stroke, vol. 35, no. 11, pp. 2529-2539, Nov. 2004.

[2] C. K. Lim, I. Chen, Z. Luo, and S. H. Yeo, "A low cost wearable wireless sensing system for upper limb home rehabilitation," in 2010 IEEE Conference on Robotics, Automation and Mechatronics, Singapore, 2010, pp. $1-8$.

[3] M. Zhang and A. A. Sawchuk, "A customizable framework of body area sensor network for rehabilitation," in 2nd International Symposium on Applied Sciences in Biomedical and Communication Technologies, Bratislava, Slovakia, 2009, pp. 1-6.

[4] H. Saito, T. Watanabe, and A. Arifin, "Ankle and knee joint angle measurements during gait with wearable sensor system for rehabilitation," in World Congress on Medical Physics and Biomedical Engineering, September 7-12, 2009, Munich, Germany, 2009, p. 506509. 